\title{
MRI-Based Classification Scheme for Degenerative Cervical Spinal Stenosis (DCSS Classification)
}

\author{
Leimert M', Hamann $\mathrm{I}^{2,3 *}$, Bostelmann $\mathrm{R}^{4,5}$, von \\ Sachsen $S^{6}$, Steinke $H^{7}$, Meixensberger $J^{6,8}$ and \\ Schackert $\mathbf{G}^{\mathbf{1}}$ \\ ${ }^{1}$ Department of Neurosurgery, Carl Gustav Carus \\ University Hospital, Technische Universität Dresden, \\ Dresden, Saxony, Germany \\ ${ }^{2}$ Department Medical Engineering, Fraunhofer Institute \\ for Machine Tools and Forming Technology, Dresden, \\ Saxony, Germany \\ ${ }^{3}$ Department of Spine Center, Asklepios Orthopädische \\ Klinik Hohwald, Neustadt i. Sa., Saxony, Germany \\ ${ }^{4}$ University Clinic Düsseldorf, Med Fac, Düsseldorf, \\ NRW, Germany \\ ${ }^{5}$ Neuro-Wirbelsäulenchirugie, Christian Hospital \\ Quakenbrueck, Germany \\ ${ }^{6}$ Innovation Center for Computer Assisted Surgery \\ (ICCAS), University of Leipzig, Leipzig, Saxony, Germany \\ ${ }^{7}$ Institute of Anatomy, University of Leipzig, Leipzig, \\ Saxony, Germany \\ ${ }^{8}$ Department of Neurosurgery, Leipzig University \\ Hospital, Leipzig, Saxony, Germany \\ *Corresponding author: Isabell Hamann, Department \\ Medical Engineering, Fraunhofer Institute for Machine \\ Tools and Forming Technology, Nöthnitzer Str. 44, 01187 \\ Dresden, Saxony, Germany
}

Department of Spine Center, Asklepios Orthopädische Klinik Hohwald, Hohwald Str. 40, 01844 Neustadt i. Sa., Saxony, Germany

Received: May 18, 2021; Accepted: June 14, 2021; Published: June 21, 2021

\section{Abbreviations}

CSM: Cervical Spondylotic Myelopathy; CT: Computed Tomography; DCSS: Degenerative Cervical Spinal Stenosis; MRI: Magnetic Resonance Imaging; OPLL: Posterior Longitudinal Ligament; SLICS: Sub-Axial Cervical Spine Injury Classification System; SPSS: Statistics Software of IBM

\section{Background}

The presence of Degenerative Cervical Spinal Stenosis (DCSS) is common in the population and becomes more widespread with increasing age [1-3]. The degeneration of the intervertebral disk and secondary degeneration of structures such as the uncovertebral joint, posterior longitudinal ligament and ligamentum flavum cause spinal cord compression and cervical myelopathy [4].

The choice of surgical treatment for DCSS and Cervical Spondylotic Myelopathy (CSM) remains controversial. The clinical practice of spinal surgery is often based on the correlation of symptoms to imaging findings, with treatment decisions rarely being based solely on imaging results. The characterization of cervical spine pathologies with Magnetic Resonance Imaging (MRI) is already

\section{Abstract}

Background: The severity of Degenerative Cervical Spinal Stenosis (DCSS) is currently assessed by determining the sagittal diameter of the spinal canal, the degree of narrowing, and the level of stenosis using magnetic resonance imaging (MRI). The aim of our study was to develop and evaluate an optimized, MRI-based classification of DCSS to support treatment decision-making.

Methods: We analyzed preoperative MRI scans of 75 patients to determine key morphological features of DCSS. Based on the features found, a classification was proposed and tested on ten representative image sets by 53 eurosurgeons to determine practicability and inter-observer reliability.

Results: DCSS extended over one (13 and $17 \%$ of pat.), two (23 and $31 \%$ of pat.) or three ( 39 and $52 \%$ of pat.). Thickening of the ligaments was observed in $71 \%$, scoliosis in $3 \%$ and grade I spondylolisthesis in $11 \%$ of the patients. The DCSS classification has three main Types (A, B, C), which differ in the extent of the stenoses and thickening of the ligaments. Each main class has 2-3 subtypes depending on the position of the stenosis (lateral/medial) and the number of stenotic segments. An additional suffix indicates the presence of concomitant pathology (spondylolisthesis, scoliosis). 26 (49\%) neurosurgeons rated the ation as useful. For the main classes, interobserver reliability was fai ( $=0.23)$. For subtype and concomitant pathology, interobserver reliability was ( $\mathrm{k}=0.14)$.

Conclusions: In the study a new classification system for degenerative spinal stenoses was developed, which combines anatomical and clinical defect characteristics. Deviations in the classification result from a strict subdivision, so hat adjustments are necessary.

Keywords: Cervical spine; Spinal canal stenosis; Classification scheme; nterobserver reliability
Austin Neurosurg Open Access - Volume 7 Issue 1 - 2021

Submit your Manuscript | www.austinpublishing group.com

Hamann et al. () All rights are reserved a clinical routine $[5,6]$ and offers an advantage in the visualization of $2 \mathrm{D}$ and $3 \mathrm{D}$ images. It reduces the risk of overlooking factors responsible for ethiopathogenesis when diagnosing with common imaging modalities such as X-rays, myelography or CT scans. MRI studies of the cervical spine are particularly important for diagnosing the cervical spine [13]. Despite the extensive use of MRI on the cervical spine, the variability inherent in MRI interpretation must be taken into account [23]. Thus, the successful application of a classification system depends largely on its reliability and the inclusion of degenerative and etiopathogenetic factors [7].

Current systems, like the Magerl comprehensive classification of thoracic and lumbar injuries [8] or the sub-axial Cervical Spine Injury Classification System (SLICS), do not help to identify the pattern and severity of injury and support treatment considerations and prognosis [8]. Degenerative changes are not considered in these established systems. The advantage of the degenerative injury classification system is that it facilitates pathomorphological uniformity by consider not only pathomorphological criteria but also the main mechanisms of injury.

The development of a DSCC classification including degenerative 
changes of the spine, based on MRI imaging, enables easier communication and support in finding a diagnosis. Especially on the view with digitization and automation of defect classifications.

\section{Methods}

\section{Key morphological features of DCSS}

The basis for the development of a classification is the identification of morphological key features.

Therefore, MRIs of the cervical spines of 75 patients (mean age $57 \pm 12$ years, age range 33-80 years) who were treated between 2009 and 2012 at the neurosurgery departments at two university hospitals were analyzed regarding their MRI data.

The MRI data were T1- and T2-weighted MRI sequences, in most cases also coronal and transverse sections of the cervical spine with a thickness of $3.5 \mathrm{~mm}$.

The scans were evaluated and analyzed by 53 neurosurgeons (more than 10 years of experience and over 100 procedures per year) based on the following aspects: multi-segment involvement of defects, osteophytes, thickness of posterior longitudinal ligaments and of ligament flava, soft disk herniation and intervertebral pathology.

\section{DSCC classification}

The classification system was established based on the key morphological characteristics determined. These were evaluated with regard to their most frequent occurrence, their most frequently occurring concomitant pathologies and their weighting from everyday clinical practice.

\section{Reliability between observers}

The reliability of a classification scheme can be determined by measuring either interobserver or intraobserver reliability [4]. To evaluate the proposed defect classification scheme on degenerative cervical spinal stenosis, ten illustrative cases were characterized by experienced German spine neurosurgeons to determine interobserver reliability and practicability. Each participant was asked to classify these cases using the developed scheme.

For statistical analysis, Fleiss' kappa [9] was calculated for the type, sub-type, and pathology of the classification (SPSS 22 for Windows, Nichols' extension module [10]). Agreement/concordance was interpreted as poor $(<0)$, slight $(0-0.2)$, fair $(0.21-0.4)$, moderate (0.41-0.6), substantial (0.61-0.8) or excellent (0.81-1) [11].

\section{Evaluation of the DSCC}

To evaluate benefit, the interviewed surgeons were asked about comprehensibility and asked to name suggestions for improvement. The evaluation was performed using a questionnaire.

\section{Results}

\section{Key morphological features of DCSS}

The evaluation of the 75 MRI scans showed that a hyperintense lesion in the spinal cord was located either near or some distance from the main stenosis.

With a proportion of $52 \%$ (39 patients), spinal stenosis spanning three or more levels were the most common form found. They were followed by mono- and bi-segmental stenosis. Thickening of the ligaments was observed in $71 \%$ of cases (53 patients), distribution between the thickening of the posterior longitudinal ligament and yellow ligaments being approximately equal the same. As expected, malposition of the vertebral bodies (scoliosis: $3 \%$, spondylolisthesis: $11 \%$ ) were one of the less common the rarer concomitant pathologies. Table 1 summarizes the MRI based on morphological findings.

The following 3 evaluation morphological features for the assessment of degenerative spinal defects achieved a special weighting:

- Extent of the stenosis (spanning one or more vertebral segments)

- $\quad$ Position of the defect (lateral or medial position)

Table 1: Stenotic segments and concomitant pathologies observed with frequency indication $(n=75)$.

\begin{tabular}{|c|c|c|c|}
\hline \multicolumn{2}{|c|}{ No. of stenotic segments (\# patients) } & \multicolumn{2}{|c|}{ Concomitant pathologies (\# patients) } \\
\hline Mono-segmental: & 13 & Thickening of the yellow ligament: \\
\hline Bi-segmental: & 23 & Thickening of the posterior longitudinal ligament \\
\hline Tri-segmental: & 39 & Scoliosis \\
\hline & & 27 \\
\hline
\end{tabular}
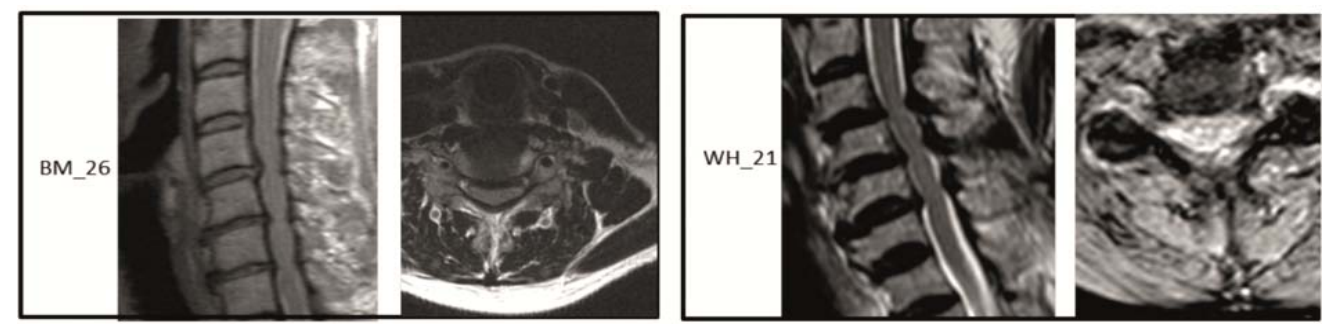

Figure 1: Two illustrative cases:

- BM26 shows a MRI T2w sag screening with a tri-segmental spinal stenosis with suspected medial and also slightly lateral compression and thickened yellow ligaments (B 1.3*), Evaluation by neurosurgeons see Table 6.

- $\quad$ WH 21 shows a MRI T2w sag screening with a tri-segmental spinal stenosis with medial compression and thickening of the yellow ligaments (B $1.3^{*}$, Evaluation by neurosurgeons see Table 5 see Table 6 
Table 2: Classification scheme for degenerative stenosis of the cervical spine.

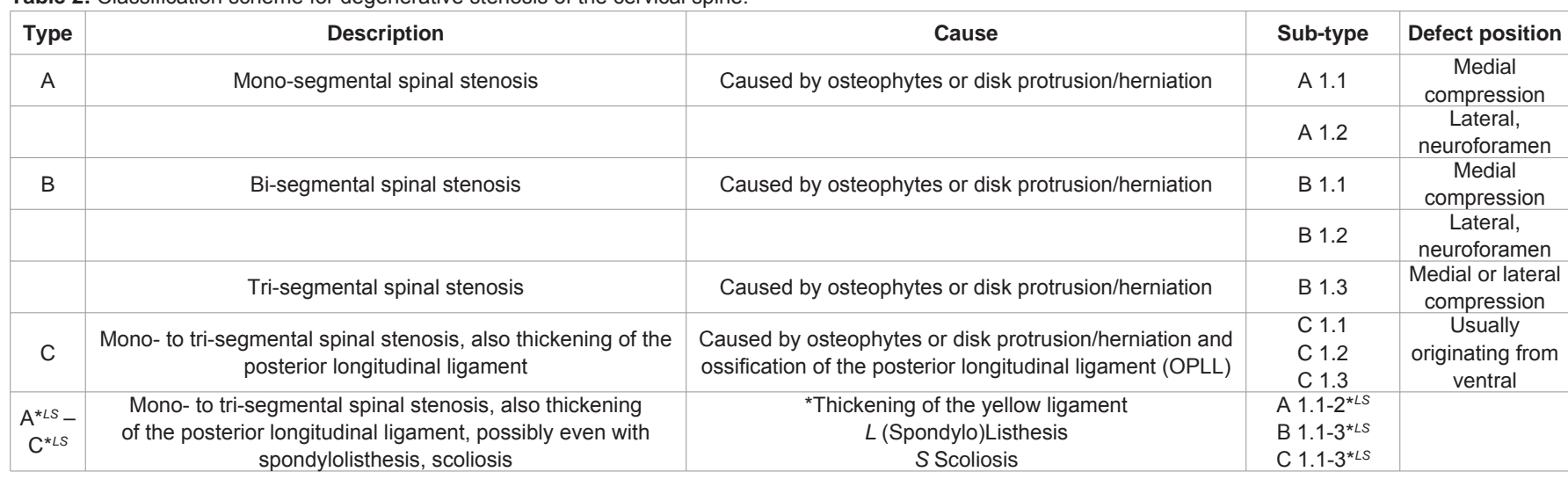

Table 3: Results of the patient-evaluation study of the new classification: Classification of 10 patients by 53 surgeons using our classification scheme (Mono: monosegmental stenosis, Bi: bi-segmental stenosis, Tri: tri-segmental stenosis, YL: yellow ligament). Figures in percent.

\begin{tabular}{|c|c|c|c|c|c|c|c|}
\hline \multirow{2}{*}{ Patients } & \multirow{2}{*}{ Mono } & \multirow{2}{*}{$\mathrm{Bi}$} & \multirow{2}{*}{ Tri } & \multirow{2}{*}{$\mathrm{OPLL}^{1}$} & \multirow{2}{*}{$\begin{array}{c}\text { Thickening } \\
\mathrm{YL}^{2}\end{array}$} & \multirow{2}{*}{$\begin{array}{c}\text { (Spondylo) } \\
\text { Listhesis }\end{array}$} & \multirow{2}{*}{ Scoliosis } \\
\hline & & & & & & & \\
\hline $\mathrm{BH} 72$ & 17 & 55 & 26 & 40 & 85 & 36 & 13 \\
\hline BK42 & 85 & 15 & - & - & 6 & 2 & - \\
\hline BK101 & 34 & 32 & 36 & 23 & 49 & 9 & - \\
\hline BM26 & 23 & 20 & 57 & 28 & 23 & 6 & - \\
\hline GF96 & 2 & 79 & 19 & 43 & 74 & 19 & 2 \\
\hline HB114 & 6 & 74 & 19 & 25 & 53 & 19 & 2 \\
\hline Jj & 2 & 8 & 91 & 51 & 49 & 8 & - \\
\hline KG34 & 47 & 26 & 17 & 6 & 17 & 45 & 4 \\
\hline sc1111 & 26 & 4 & 11 & 15 & 15 & 9 & 38 \\
\hline WH21 & 8 & 32 & 57 & 26 & 66 & 42 & 8 \\
\hline
\end{tabular}

${ }^{1}$ Posterior longitudinal ligament

${ }^{2}$ Yellow ligament

Table 4: Cross tabulation: Ratings by number of previously performed surgeries by a surgeon.

\begin{tabular}{|c|c|c|c|c|c|}
\hline & & \multicolumn{3}{|c|}{ Professional experience (number of surgeries) } & \multirow[t]{2}{*}{ Total sum } \\
\hline \multirow{4}{*}{ Classification useful? } & & $<50$ & $50-100$ & $>100$ & \\
\hline & Yes & 7 & 1 & 18 & 26 \\
\hline & No & 3 & 8 & 16 & 27 \\
\hline & & 10 & 9 & 34 & 53 \\
\hline
\end{tabular}

- Concomitant pathology (thickened ligaments, olisthesis and scoliosis (Figure 1).

Figure 1 shows sagittal and transversal views of two illustrative cases.

\section{DSCC-classification}

Based on the stenotic and concomitant pathologies identified, a classification scheme was defined (Table 2). Classification Type A and $\mathrm{B}$ ignore the thickening of the ligaments. Type $\mathrm{C}$ defines stenosis with a thickening of the posterior longitudinal ligament. For each type, two sub-types were defined to describe the position of the stenosis, type B also having a third sub-type to describe tri-segmental stenosis. Type C sub-types do not indicate the position of the defect (in contrast to types A and B), but instead classify the number of stenotic segments. Each of the defined sub-types is further classified on the presence of concomitant pathologies (spondylolisthesis or scoliosis).

\section{Reliability between observers}

All 10 sets of scans were rated completely by 21 surgeons; 25 surgeons rated 9 out of 10 sets, 6 rated 8 and 1 surgeon rated only 7 sets. Interobserver reliability was calculated based on the classification of the 10 cases (Table 3). Fair agreement was recorded concerning type of stenosis ( $\kappa$-value of 0.23 ). However, interobserver reliability decreases to slight agreement (k-0.14) regarding classification of spinal canal stenosis down to sub-type and concomitant pathology.

\section{Evaluation of the DSCC}

53 of the called 166 neurosurgeons rated the patients' scans using our defect classification. The neurosurgeons had varying levels of experience (Table 4), with a mean experience as a neurosurgeon being $11 \pm 9$ years. The majority of participants had treated over 100 cases of spinal canal stenosis. Our cervical stenosis DCSS - classification scheme was rated as understandable by $79 \%$ and useful by nearly half 
Table 5: Participants' comments about the MRI-based defect classification scheme.

\begin{tabular}{|c|c|}
\hline- & Classification not stringent or logical within itself à too complicated à too confusing (2) \\
\hline- & Clinical aspects/criteria/complaints ignored (15) \\
\hline- & No combination of medial and lateral compression (6) \\
\hline- & Neurological symptoms/deficits should be included (5) \\
\hline- & Indication of myelopathy (5) \\
\hline- & Ignores distally affected segments (4) \\
\hline- & Distinction required between thickening of $O P L L^{1}-$ own pathology (2) \\
\hline- & Functional images should be included - may be important for proof of listhesis (2) \\
\hline- & Classification should be changed into $A B C$ as the $A B C$ classification of Magerl is already well established (1) \\
\hline- & $A, B, C$ for number of segments (1) \\
\hline- & ${ }^{\star 2}, L^{3}, S^{4}$ add-ons provide little additional information à should be omitted (1) \\
\hline- & Degree of stenosis should be indicated (1) \\
\hline- & Definition of stenosis (1) \\
\hline- & No evaluation of kyphosis - important for potential prosthesis (1) \\
\hline- & All $S$ in group $C$, if necessary also all $L$ in group $C(1)$ \\
\hline- & Differentiation of neuroforamina (unilateral/bilateral) (1) \\
\hline- & Related multi-segmental pathology or possibly skip lesion should be considered (1) \\
\hline- & Sub-classification should be expanded (adjacent segment versus non-adjacent segments) (1) \\
\hline- & B1-3 with OPLL and leave out C (1) \\
\hline- & Bone quality should be included (1) \\
\hline
\end{tabular}

\section{${ }^{1}$ Posterior longitudinal ligament}

${ }^{2}$ Thickening of the yellow ligament

${ }^{3}$ (Spondylo) Listesis

${ }^{4}$ Scoliosis

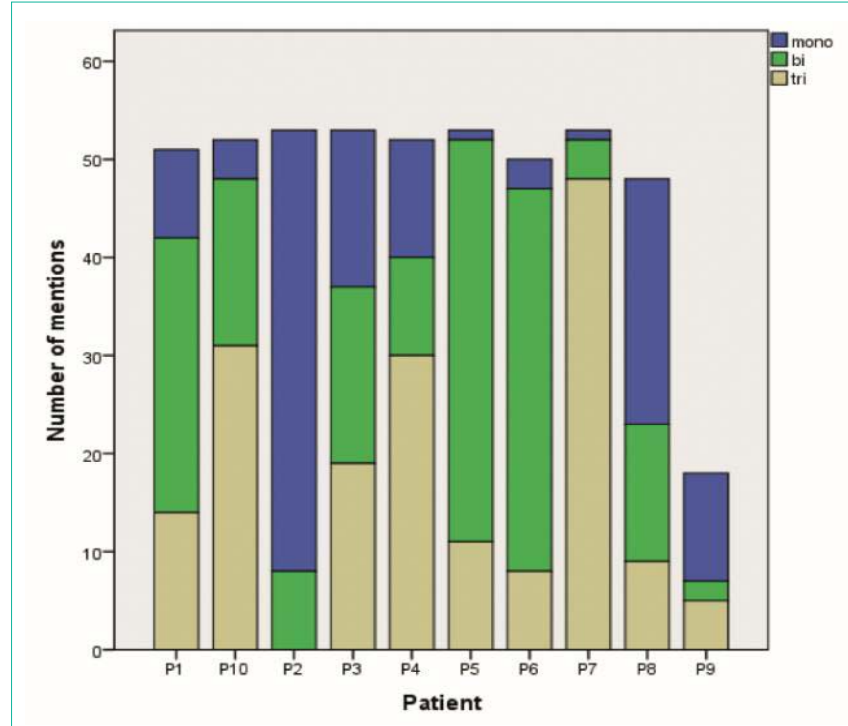

Figure 2: Use of classification overview.

(49\%). Table 4 shows the correlation between work experience and assessment of the benefit of the classification scheme. The analysis of the data in Table 4 shows the distribution of the expert groups between supporters and skeptics. Table 5 contains comments on the classification scheme for morphological defects.

\section{Application on an example}

Patient 3 (BM26): 55 years old, female (Figure 1).

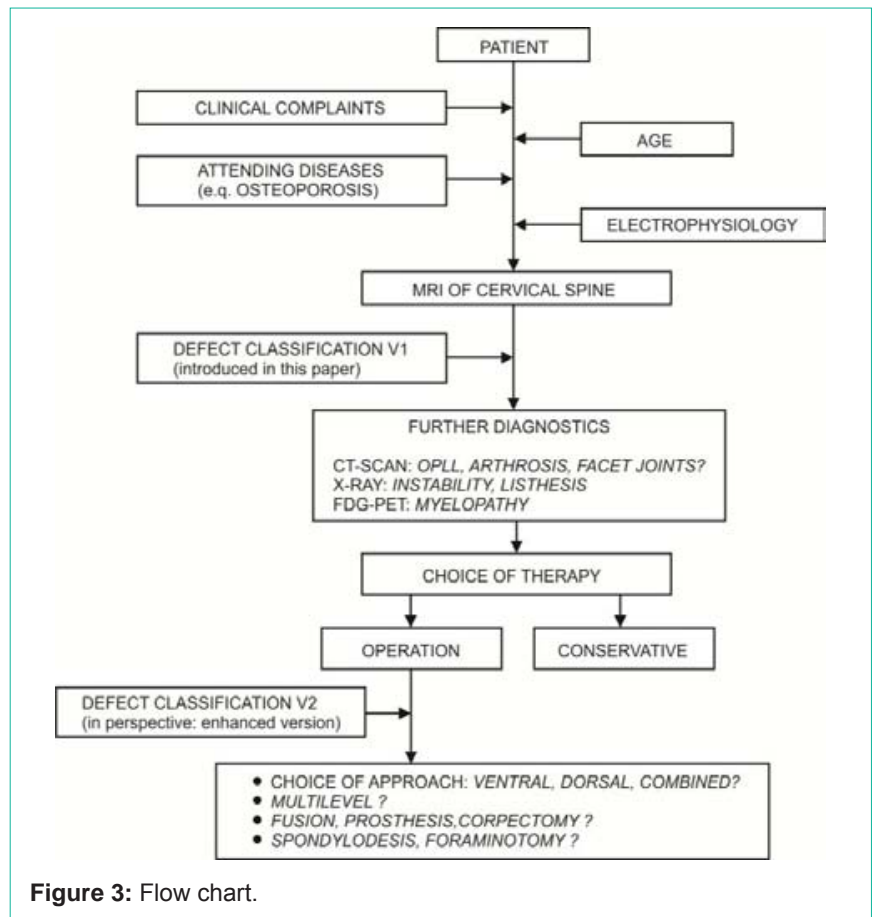

Description: A very straight cervical spine doubtless with a constitutionally narrow cervical spinal canal, so that developing pathologies may have significantly more dramatic clinical effects and stenosis; cervical vertebrae 4-7: probably myelopathy signal near 
Table 6: The table shows the three most frequent answers of the evaluation of patient BM_26 and WH_21 by the new classification according to type, sub-type and accompanying pathology.

\begin{tabular}{|c|c|c|c|c|c|c|}
\hline BM_26 & & $\mathbf{n}(\%)$ & & $\mathbf{n}(\%)$ & & $\mathbf{n}(\%)$ \\
\hline Type & B & $28(54)$ & C & $15(29)$ & A & $9(17)$ \\
\hline Sub-type & B 1.3 & $19(37)$ & C 1.3 & $11(21)$ & A 1.1 & $8(15)$ \\
\hline Concomitant pathology & $* 1$ & $10(19)$ & $* \mathrm{~L}^{2}$ & $2(4)$ & $\mathrm{L}$ & $1(2)$ \\
\hline WH_21 & & $\mathrm{n}(\%)$ & & $\mathrm{n}(\%)$ & & $\mathrm{n}(\%)$ \\
\hline Type & $\mathrm{B}$ & $35(67)$ & $\mathrm{C}$ & $14(27)$ & $\mathrm{A}$ & $3(6)$ \\
\hline Sub-type & $\mathrm{B} .1 .3$ & $21(40)$ & $\mathrm{B} .1 .1$ & $10(19)$ & $\mathrm{C} 1.3$ & $10(19)$ \\
\hline Concomitant pathology & $*$ & $18(35)$ & $* \mathrm{~L}$ & $15(29)$ & $* \mathrm{LS}^{3}$ & $2(4)$ \\
\hline
\end{tabular}

${ }^{1}$ Thickening of the yellow ligament

${ }^{2}$ (Spondylo) Listesis

${ }^{3}$ Scoliosis

cervical vertebrae 5/6 (M); additional CT scan necessary for a definite assessment of the posterior longitudinal ligament, which may appear to be thickened (as in this case) since the height of the intervertebral disks is decreasing and the ligaments are starting to develop folds.

Evaluation based on DCSS: 28 (54\%) of the surveyed neurosurgeons classified the example in Type B, 15 (29\%) in Type C and $9(17 \%)$ in A. As sub-types were chosen with a total of 19 votes (37\%) B 1.3, with 11 (21\%) type C 1.3 and with 8 (15\%) A 1.1. 10 (19\%) reported a thickening of the yellow ligament, 2 (4\%) a thickening of the yellow ligament and one surgeon (2\%) reported an accompanying pathology with only one olisthesis.

\section{Discussion}

Based on the key morphological features identified - Extent of the stenosis (spanning one or more vertebral segments), Position of the defect (lateral or medial position) and Concomitant pathology (thickened ligaments, olisthesis and scoliosis.) - a degenerative classification system was developed, which was rated as understandable by $79 \%$ and useful by nearly half (49\%).

Surgical treatment of DCSS, CSM or radiculopathy due to cervical stenosis has increased significantly in all industrialized countries, especially among those aged 75-80 [12]. DCSS and CSM are common causes of neurological morbidity, they potentially lead to a significant deterioration in quality of life [13]. In spine surgery, it is therefore important to correlate the symptoms with the imaging findings. Therefore, a review and enhancement of the current classification systems is necessary to support diagnostic strategies.

\section{Key morphological features of DCSS}

An important requirement for the development of classification systems for the stenosis of the spine is that anatomical defect characteristics and clinical complaints are equally taken into account and possible correlations are highlighted [14].

Although MRI is a widely used tool for the evaluation of degenerative disease of the cervical spine and the visualization of pathological alterations, which may lead to compression of the cervical spinal cord and consequently CSM, there is surprisingly disagreement about the surgical treatment of this compression of the cervical cord [15]. According to Chapman et al., the diagnostic components anatomy, biomechanics, clinical status and severity form the basis for any classification of spinal defects [14]. The classification of defects therefore provides an important basis for comparing different therapeutic concepts and studies. Currently, defect classification systems based on the sagittal diameter of the spinal canal [16-18] or the degree of narrowing and the position of the stenosis [19] in radiological images are used to describe stenosis of the cervical spine. Other anatomical defect features that can be identified by MRI and which could influence the therapeutic decision are neglected.

It was shown that especially the extent of the stenosis is a key factor for the evaluation of DSCC and has a high weighting in an evaluation. In this context and as a result of this, the position of the defects and the concomitant pathology are important in order to weigh up statements about therapy options. The additional defect characteristics identified in this study provide an assistance for the assessment of degenerative diseases and their therapy.

\section{DSCC-classification}

The primary aim of developing disease-specific DCSS classification systems is to help physicians diagnose and select the best treatment strategy. This more sufficiently can be done with complex classifications. Moreover, a classification scheme enables defects to be classified within a more comprehensive and broader framework, which in turn supports communication with peers, guideline management and related research activities, e.g. the development of defect-specific treatment strategies [14]. In the future, this will serve as an approach for an automated computer-based classification system based on MRI imaging for ultimately individualized medicine and implant design.

The current classification systems according to Magerl and the subaxial system for the cervical spine support the treating physician in the diagnosis and thus in the treatment strategy. To subdivide the injuries a classic 3-3-3 AO scheme is used. The focus of these systems are on the evaluation of fracture formation, fracture type and position of the injury [8]. The systems neglect the degenerative aspects of the ligaments, such as thickening of the ligaments and scoliosis. This is one of the main differences between traumatological or orthopedic scales and the here presented degenerative scale which was introduce here after a reviewing this classification by 53 experienced neurosurgeons.

Considering degenerative aspects, a simple subdivision such as the 3-3-3 scheme of $\mathrm{AO}$ fracture classification is unsuitable. Therefore, the DSCC classification focuses on the number of injured vertebrae involved, the defect localization and the accompanying pathologies such as thickening of the yellow ligament, olisthesis and scoliosis. Figure 2 shows an overview of the use and workflow of classification. Clinical aspects were incorporated that are associated with degenerative changes, so that they give the user a new perspective on the injury.

\section{Reliability between observers}

The evaluation of the 10 representative cases using the DSCC classification showed a very inhomogeneous classification.

Tables 6 and 7 show the most frequently cited classification suggestions for two of the ten patients used in the study. The responses are organized by type, sub-type and concomitant pathology. Since there were only three alternatives when selecting types, the sum of all answers is always $100 \%$. In the case of sub-types 
and concomitant pathology, only the three most frequently given responses are listed, so the sum of all answers is not always $100 \%$. All answers are given from left to right in order of frequency. The calculation of the interobserver reliability fair resulted in a K-value of 0.23 . For the subtype and accompanying pathology the reliability between observers was low $(\mathrm{k}=0.14)$. While developing the MRIbased classification, the enormous discrepancy in the evaluation of MRI scans depending on surgical experience was remarkable. For instance, patient BM26 suffered from high-grade three-level cervical canal stenosis, yet $43 \%$ of the surgeons (the younger, less experienced ones) ignored this problem (Figure 1).

The DSCC system involve a total of 56 different subclassifications, 52 of which appeared during the classification study. The strict subdivision of the classification scheme is thought to be the main cause of the low agreement rate when comparing the classifications performed by 53 surgeons. A similarly strict subdivision can also be found in Magerl's description of traumatic spinal defects [17], for which a total of 55 different sub-classifications are possible. The interobserver reliability of the Magerl classification (14 spinal injuries were classified by 21 clinics) assessed by Blauth [3] shows only moderate agreement, which can also be attributed to the complex grading of defect characteristics. When assessing the Magerl classification, a $\kappa$-value of 0.33 was determined for type classification (A, B, C), 0.27 for the group division, 0.23 for sub-group 1 and 0.21 for sub-group 2 . The interobserver reliability for the classification scheme developed by Magerl can be rated as fair for all levels. However, good interobserver reliability is only assumed as of a $\mathrm{k}$-value of 0.61 . The results of the interobserver reliability of the classification of stenosis of the cervical spinal and the classification system developed by Magerl underline the challenge of creating a practical classification scheme for the description of defects. The results presented in this article show that the rate of agreement regarding the classification of spinal defects by multiple physicians decreases as the classification structure increases. Chapman et al. state that if defect classification systems are to be clinically beneficial, they must be simple and easy to remember and recommend treatment strategies based on the identified severity of the defect.

Another reason for the different classification can be, besides experience, the subjective impression of each individual surgeon. Inconsistent definitions of types and subtypes increases the effect and impede a consistent, reproducible evaluation of patients. When reading imaging reports or discussing MRI findings with patients and colleagues about clinical findings and the typical complaints of the individual patient, all physicians should be aware of inconsistencies in the interpretation of MRI findings.

The quality of the MRI scans is the main requirement for interobserver reliability. For example, the MRI scans of patients KG34 and SC1111 were of reduced quality with very thick axial sections, accounting for the lack of accordance. Surprisingly, however, even high-quality MRI scans with very thin axial sections did not necessarily lead to high accordance.

Furthermore, it is unclear why only $36 \%$ saw the clear C4/ C5 spondylolisthesis in patient BH72. 45\% of all colleagues saw a spondylolisthesis in patient KG34, where in fact a retrolisthesis may well be seen.

\section{Evaluation of the DSCC}

The assessment of the MRI-based classification of stenosis of the cervical spinal canal clearly shows the great interest of surgeons in a systematic description of the stenosis of the cervical spinal and encourages future work in this area.

MRI is usually used to evaluate degenerative conditions of the cervical spine. However, the lack of consistency in interpreting and reporting of degenerative findings from cervical MRI on patients with DCSS and CSM, which influence treatment decisions, was not well rated [7].

A distinct and systematic description is also important for the development of individualized medical technology, such as the adaptation of screw or vertebral body implants for the cervical spine. Researchers and medical technology engineers work closely with medical teams and are therefore dependent on consistent commutation. Moreover, a classification scheme enables defects to be classified within a more comprehensive and broader framework, which in turn supports communication with peers, guideline management and related research activities, e.g. the development of defect-specific treatment strategies [14]. The implant development is based on the patient-specific data. It therefore offers great potential to adapt the implants to the classification types and their treatment and to increase the success of the treatment. In addition, the wellknown surgical complication rate in multilevel cases underscores the importance of optimizing the surgical approach for each patient and determining the optimal surgical approach $[20,21]$

It is therefore confusing why there are no established guidelines for treating patients with CSS and CSM. Judging by our questionnaire, we may have suggested an adequate solution to this problem that takes anatomical defect features and clinical complaints equally into account and shows possible connections. This result is in accord with the comments of the participants and should be consider for further schemes.

A strength of the presented study is the participation of more than 50 neurosurgeons, the specific view on the pathologies and the result that nearly half the participants found our classification scheme useful. The major reason for surgeons who dismissed our classification scheme is the purely morphological focus without considering clinical complaints and that only neurosurgeons were involved, not radiologists, neuroradiologists or orthopedic spine surgeons. They might interpret these cervical MRI studies differently, which could be explored in future studies [7].

Nevertheless, our results underline the importance of correlating imaging findings with clinical findings and combining them with our defect classification scheme when providing patient care.

In the next development step, the most important clinical complaints and the changes in the long-term course depending on the respective procedure will be recorded and included into the classification scheme. Furthermore, the terminology of the previously developed draft is to be revised in order to clearly differentiate it from the Magerl classification and to ensure consistent coding of the subtypes. In particular, the description of the defect, which currently consists of three levels (type, subtype, concomitant pathologies), is to be reduced to one or two levels in order to enable rapid classification. 
The classification of all visible anatomical changes in MRI scans and combination within the same system is a new diagnostic approach to the treatment of degenerative cervical spine diseases.

Current programs for evaluating image data, masking defects and segmentation of organs are standard surgical tools for the diagnosis and therapy of spinal diseases and can be enhanced by the classification [22]. A widened evaluation of radiological data as done here allows a more complex analysis that classification based on one segment or the situation of the bones and offers a decisive approach to combine and support of diagnostic, treatment and surgical strategies. [17-19]. Here, the image-based classification for the calculation of solutions and evaluation protocols offers a decisive approach to combine and support of diagnostic, treatment and surgical strategies.

\section{Limitations}

One clear limitation was the varying quality of the MRI scans, although this reflects the reality of surgical routine. Other studies should focus on new, high-quality MRI scans, as well as involve functional X-ray images and CT scans.

Clinical aspects such as radiculopathy and myelopathy were not considered in this study. For a simple and understandable classification, the extension by evaluation of adjacent segments and bone quality was committed. In further studies, these should be considered and included in order to be able to make stronger statements about therapy options.

No distinction was made between thickening of OPLL as a separate pathology.

Further investigations to refine and increase the complexity of DSCC, which were not considered in this work, include aspects such as medial and lateral compression, neurological symptoms/deficits and the degree of scoliosis/kyphosis.

\section{Conclusions}

A new classification system for degenerative cervical spine is proposed. Regarding the treatment of the patients in our groups, we recommend choosing the surgical approach based on our DCSS classification system along with other clinical und radiographically parameters. Its limitations are the non-involvement of clinical complaints, electrophysiological data and dynamic radiographs.

\section{Funding}

This work was funded by the European Regional Development Fund (ERDF) and the Free State of Saxony under measures supporting the technology sector (Grant No. 100096769).

\section{Informed Consent}

Additional informed consent was obtained from all individual participants for whom identifying information is included in this article.

\section{Author's Contributions}

All authors read and approved the manuscript.

\section{Acknowledgment}

Many thanks to M.D. Johannes Gerber and Mr. Abbey who helped with proofreading.

\section{References}

1. Bernhardt M, Hynes RA, Blume HW. White Cervical spondylotic myelopathy. In: JBJS. 1993; 75: 119-128.

2. Ferguson RJ, Caplan LR. Cervical spondylitic myelopathy. Neurol Clin. 1985; 3: 373-382.

3. Ogino H, Tada K, Okada K, Yonenobu K, Yamamoto T, Ono K, et al. Canal Diameter, Anteroposterior Compression Ratio, and Spondylotic Myelopathy of the Cervical Spine. In: Spine.1983; $8: 1-15$.

4. Edwards CC, Riew KD, Anderson PA, Hilibrand AS, Vaccaro AF. Cervical myelopathy. The Spine Journal. 2003; 3: 68-81.

5. Schmidt KI, Viera J, Reith W. Degenerative Erkrankungen der Wirbelsäule (Degenerative diseases of the spine). Radiologe. 2011; 51: 779-783.

6. Fu MC, Buerba RA, Neway WE, Brown JE, Trivedi M, Lischuk AW, et al. Three-Dimensional Isotropic MRI of the Cervical Spine: A Diagnostic Comparison With Conventional MRI. Clin Spine Surg. 2016; 29: 66-71.

7. Fu MC, Webb ML, Buerba RA, Neway WE, Brown JE, Trivedi M, et al. Comparison of agreement of cervical spine degenerative pathology findings in magnetic resonance imaging studies. Spine J. 2016; 16: 42-48.

8. Magerl F, Aebi M, Gertzbein SD, Harms J, Nazarian S. A comprehensive classification of thoracic and lumbar injuries. Eur Spine J. 1994; 3: 184-201.

9. Fleiss JL, Levin B, Paik MC, Shewart WA, Wilks SS. Statistical Methods for Rates and Proportions. John Wiley \& Sons, Inc, Hoboken, NJ, USA. 2003.

10. IBM. Compute Fleiss Multi-Rater Kappa Statistics. IBM. 2014.

11. Landis JR, Koch GG. The Measurement of Observer Agreement for Categorical Data. Biometrics. 1977; 33: 159-174.

12. Kristiansen J-A, Balteskard L, Slettebø H, Nygaard ØP, Lied B, Kolstad F, et al. The use of surgery for cervical degenerative disease in Norway in the period 2008-2014: A population-based study of 6511 procedures. Acta Neurochir (Wien). 2016; 158: 969-974.

13. Blauth M, Bastian L, Knop C, Lange U, Tusch G. Interobserverreliabilität bei der Klassifikation von thorakolumbalen Wirbelsäulenverletzungen (Interobserver reliability in the classification of thoraco-lumbar spinal injuries). Orthopade. 1999; 28: 662-681.

14. Chapman JR, Norvell DC, Dettori JR. Spine classifications and severity measures. AO spine. Thieme, Stuttgart, New York. 2009.

15. Bilbao G, Duart M, Aurrecoechea JJ, Pomposo I, Igartua A, Catalán G, et al. Surgical results and complications in a series of 71 consecutive cervical spondylotic corpectomies. Acta Neurochir (Wien). 2010; 152: 1155-1163.

16. Boden SD, Dodge LD, Bohlman HH, Rechtine GR. Rheumatoid arthritis of the cervical spine. A long-term analysis with predictors of paralysis and recovery. J Bone Joint Surg Am. 1993; 75: 1282-1297.

17. Edwards WC, LaRocca H. The developmental segmental sagittal diameter of the cervical spinal canal in patients with cervical spondylosis. Spine. 1983; 8: 20-27.

18. Pavlov H, Torg JS, Robie B, Jahre C. Cervical spinal stenosis: determination with vertebral body ratio method. Radiology. 1987; 164: 771-775.

19. Muhle C, Metzner J, Weinert D, Falliner A, Brinkmann G, Mehdorn MH, et al. Classification system based on kinematic MR imaging in cervical spondylitic myelopathy. AJNR Am J Neuroradiol 1998; 19: 1763-1771.

20. Edwards CC, Heller JG, Murakami H. Corpectomy versus laminoplasty for multilevel cervical myelopathy: an independent matched-cohort analysis. Spine. 2002; 27: 1168-1175.

21. Sampath $P$, Bendebba M, Davis JD, Ducker TB. Outcome of patients treated for cervical myelopathy. A prospective, multicenter study with independent clinical review. Spine. 2000; 25: 670-676.

22. Free Software Foundation I. DICOM Validation Toolkit. DVTk, Boston, MA 02110-1301 USA. 2018. 
23. Speciale AC, Pietrobon R, Urban CW, Richardson WJ, Helms CA, Major Net al. Observer variability in assessing lumbar spinal stenosis severity on magnetic resonance imaging and its relation to cross-sectional spinal canal area. Spine. 2002; 27: 1082-1086. 